\title{
Blockchains, Property Rights and Health Technology Assessment in the Pharmaceutical and Device(s) Industries
}

Paul C Langley, PhD ${ }^{1}$ Robert E Martin, MS RPh ${ }^{2}$

${ }^{1}$ Adjunct Professor, College of Pharmacy, University of Minnesota

${ }^{2}$ Folsom CA

\begin{abstract}
Ongoing concerns with the security of health information, both from the perspective of the individual patient as well as health systems has led to increased attention being given to the potential role of blockchain technology in the secure storage of health information through encryption, the integration of diverse health record systems and the vesting of property and access rights to health data in the patient. While the security offered by blockchain technology has long been recognized in the finance sector with the emergence of a range of cryptocurrencies as a medium of exchange and store of value, demonstrating the value of blockchain technology in health management and health technology assessment has yet to be achieved. In this commentary, a number of questions are raised as to the potential value offered by blockchain technology as a complement to existing electronic medical record systems. Chief among these are: (i) the allocation of property rights as a necessary precondition for blockchain uptake; (ii) access and incentives for active as opposed to passive blockchain membership; (iii) monetization of blockchain access; (iv) capturing data from within the blockchain and the possibility of value added data; (v) the potential for blockchain platforms in formulary evaluations; (vi) the blockchain as a managed market for health data; and (vii) the role of intermediation in blockchain management.
\end{abstract}

Keywords: blockchain technology, blockchains in health, barriers to blockchain implementation, health data property rights

\section{Introduction}

A robust and comprehensive evidence base is critical in health care decision-making. The absence of a comprehensive evidence base has been recognized as a key element in explaining why the US health care system, comprising over $20 \%$ of the US economy, not only delivers health care at a higher per capita cost than in comparable developed economies but delivers outcomes for the population that lag behind those achieved in countries that spend far less. Shortfalls in the evidence base are seen in a number of areas. Two of the most significant are: (i) a lack of access on the part of health system decision makers to information that would enable them to monitor and validate, including replicate, the claims made by manufacturers for pharmaceutical products and devices in target patient populations; and (ii) limited access to information by patients that would enable them to avoid choosing unnecessarily high cost providers in favor of providers who offer high value and cost-effective treatment.

The need for an improved evidence base has been addressed by a number of commentaries published in INNOVATIONS in Pharmacy over the past 2 years ${ }^{1}$. The most recent commentary pointed to the importance of distinguishing 'evidence' from information' in health system decision

Corresponding author: Paul C Langley, PhD

Adjunct Professor

College of Pharmacy University of Minnesota

Director, Maimon Research LLC

5061 North Apache Hills Trail, Tucson, AZ 85750

Email: langley@maimonresearch.com making ${ }^{2}$. Rather than health systems relying on modeled claims for comparative product performance, the focus should be on establishing platforms to support protocol driven claims assessments as part of the formulary evaluation process. It was proposed that applications of blockchain technology might provide such a platform. The purpose of this commentary is to explore in greater depth the potential benefits of blockchains as health technology assessment platforms.

\section{The Role of Blockchains}

From a healthcare perspective, the role of a blockchain is to overcome the inefficiencies generated by a fragmented health information system. Substantial barriers exist to the sharing of information between health providers, hospitals, pharmacies and laboratories. This is exemplified by the lack of interoperability between the variety of electronic medical record (EMR) systems, and disparate lab, pharmacy and radiology data reporting systems. At the same time, there are increasing concerns over the privacy of health care information, its susceptibility to hacking and the uncertainties that attach to the ownership of healthcare data. As to this last point the issue is not just the ability of third parties to hack data systems but the apparent willingness of health systems and data collection agencies to sell patient data which is often poorly de-identified, to third parties without seeking patient consent.

Establishing a blockchain system that stores medical records and vests the property rights to those records held or assigned to the patient, offers a potentially viable next step. A private, decentralized healthcare non-ethereum blockchain offers a secure encrypted environment for their healthcare data, giving 
the patient sole control over access/ownership. This opens the opportunity for the patient not only to share these data with selected providers, but to monetize their healthcare data for research and other applications by third parties through tokenization.

With property rights vested in the patient, the patient as gatekeeper has the opportunity, presumably with the support of market intermediaries, to realize the value implicit in their healthcare data that, at the moment, is expropriated by insurance companies and other third party data vendors. One example would be the ownership of DNA profiles and ability to monetize these data through selected access rather than, often without the knowledge of the patient, having their data sold to third parties. Drug manufacturers, for example, are a ready market for DNA profiles in drug development. With vested property rights, the patient or the patient's agent is in a position to establish a market price for these data, and for the owner to be reimbursed through tokenization.

A healthcare blockchain is a private (for HIPAA requirements) decentralized distributed transaction ledger where each block represents a real-time transaction between the patient and a healthcare provider. Each transaction is linked, creating a record embodying cryptographic techniques for full transparency and decentralization, creating a trust-less consensus rather than a centralized authority overseeing the data. Each transaction is validated through a process known as mining which validates the transaction or block in a network through the application of complex algorithms to prove and validate the correctness of a transaction, storing the data in a 'block'. Once validated, timestamped and added to the blockchain, the transaction cannot be revisited for subsequent modification (i.e., immutable). All health data in the blockchain are indexed, linking the health data to a unique identifier for the patient. Health data from wearable devices and mobile applications can be sorted and indexed accordingly. Aggregated data from all providers can be captured through query or search protocols driven by data dictionaries.

All requests for data access have to be approved by the patient, via "opt-in" or "opt-out" choices. There are no 'backdoors' to the system and the likelihood of hacking or unauthorized data access is essentially impossible. The patient is at liberty, therefore, to approve or deny access and to determine which data elements or transactions are open. This allows for the monetization of access permissions. One approach is to utilize utility tokens within the system where smart contracts for data provision can be established at the token 'price' for those data elements. Tokens can be exchanged for cryptocurrency which can then be stored or exchanged for fiat dollars in the cryptocurrency markets.
Of course, the ability of patients to monetize their data through tokenization, presupposes a market for those data and intermediaries that negotiate on behalf of target patient groups. This raises the issue of whether blockchain healthcare platforms are seen as essentially passive storage devices or as an active asset that patients can use to generate income. In the former case, it is not clear who would create and manage the blockchain and whether patients would be required to 'opt into' the blockchain. Presumably, the blockchain would be managed by a commercial entity. The presence of a commercially managed blockchain may eliminate the need for patients to subscribe to participate in the blockchain. Rather, the owners could act as intermediaries to monetize the blockchain data through identifying and seeking permissions from target patient groups for data access. Under this scenario, the blockchain owner would encourage patients to participate actively in data access, encouraging (as noted below) value added data assembly to enhance the returns to the owners and participating patients via tokenization.

\section{Value Added and Blockchains}

There are a number of opportunities open to blockchain vendors to develop distributed applications (Dapps) based on their various platforms. For these to be considered as viable rather than purely speculative, they need to be evidence based, driven by protocols with the blockchain management providing technical and clinical support.

A range of Dapps could be considered, a number of which would meet unmet medical needs in the area of health technology assessment. Potential applications could include:

- Claims assessment protocols for new products and devices (see below)

- Tracking and response monitoring for target patient populations

- Clinical trials (focus on replication of claims)

- Generic and disease specific quality of life assessment

- $\quad$ Satisfaction with care evaluations

- Patient reported outcomes assessments in specific disease states

- Risk assessments for medication abuse

- $\quad$ Risk assessment for adherence and persistence with therapy

- $\quad$ Compliance tracking

- Wellness and behavioral health incentives (see below)

Specific applications could be directed to claims made by manufacturers for their products. All too frequently, the evidence base for clinical claims is limited. For formulary committees to accept claims, they may quite reasonably ask 
manufacturers to utilize a blockchain platform to report back on claims made and those for cost-effectiveness.

As an extension, formulary committees could ask manufacturers to undertake comparative claims assessments as part of disease area and therapeutic class reviews. Again, the blockchain platform could provide the necessary structure to support both prospective as well as retrospective comparative studies (see below).

\section{Blockchains and Electronic Medical Records}

It should be made clear that a blockchain platform is not a substitute for an EMR. While it would be possible to program systems such as Epic as a blockchain system, the question of property rights is probably best resolved by a blockchain that sits alongside and interacts with an EMR platform. After all, if a patient moves between providers and health systems, or one country to another, questions will arise as to the ongoing ability of the patient's EMR blockchain to talk to the 'new' medical record system, and 'seamlessly' continue to capture and store records. This would apply both to commercial EMR platforms as well as to software systems such as the CMS Medicare MyMedicare.gov personalized website.

Once a blockchain is in place, and sufficient patients have been recruited to the blockchain, a number of opportunities as noted above open up for value added activities. One opportunity is for the patient to 'sell' data elements that are captured from the EMR. This would require the application of search algorithms together with data dictionaries to identify required data elements. Unfortunately, searching EMRs is not as straightforward as experience with the University of Michigan EMERSE search engine has demonstrated ${ }^{3}$. A major concern must be the quality of the data in terms of incompleteness, inconsistency and inaccuracy. There is not the ability to apply a big data model which simply crunches millions of administrative claims records.

Rather than just providing access to data captured and integrated from the patient's various provider EMRs the ability, as noted above, to utilize the blockchain platform as a vehicle for targeting distributed applications, avoids the pitfalls associated with developing search engines for EMRs. Certainly key data elements could be targeted such as ICD-10CM, CPT and NDC codes, but these would be seen as adjunct elements to identify patients prior to soliciting their interest in specific applications.

The choice of structured questionnaires and reports completed at each practice visit by the patient and the physician would support data interrogation through a data dictionary. This presents the opportunity for integrating patient reported outcomes instruments (PROs) in structured questionnaires and reports. Reports could be prepared at the individual patient level summarizing therapy response over baseline, together with reports to capture the distribution of therapy responses within the targeted patient group.

\section{Formulary Evaluations}

Over the past two years a number of commentaries have been published in INNOVATIONS in Pharmacy pointing to the difficulties of establishing and replicating RCT outcomes claims made by manufacturers for product performance across treating environments. Frequently, formulary committees have to rely upon clinical and cost-effectiveness claims extrapolated from pivotal randomized trials. Not only do these trials often lack external validity in terms of targeted treatment populations, but the limited duration of the trial doesn't match real-world evidence treatment cycles.

The need for manufacturers to evaluate and replicate claims for their products in real world evidence (RWE) environments has been addressed in the recently published Minnesota proposed guidelines for formulary evaluation (version2) 4 . Formulary committees would require manufacturers, prior to final pricing and tier position decisions, to undertake a prospective observational study, to a protocol agreed between the parties. The protocol should allow feedback to the formulary committee in a short-term, meaningful time frame. This allows a full review of the product and its claims in targeted treatment populations to be realized. A blockchain platform is ideally suited to this purpose

\section{Blockchains and Registries}

Over the past 20 years, considerable resources have been devoted to the establishment of registries to track patient outcomes. Unfortunately, patients seldom benefit from registry participation as property rights are typically transferred and vested in the registry owner. As noted in a previous commentary, while a blockchain is not a disease registry or a single use platform for a specific intervention, it is important to note the synergies between disease registries as platforms and a blockchain as a platform ${ }^{1}$. A blockchain not only supports a registry through protocols to generate structured healthcare data, but the incentives built into the blockchain model with assigned property rights have the potential to enhance patient retention in the registry.

The blockchain can be seen as a registry umbrella. Rather than creating registries as a response to data limitations, the blockchain provides the framework for tracking patients within targeted patient populations. Any number of registry analogs with built in incentives for patient participation can potentially be supported. The added advantage, of course is that the blockchain as the repository of the entire medical record offers opportunities to link specific applications to track response to therapy to that record. 


\section{Wellness and Behavioral Incentives}

A further potential application of the blockchain model is to support programs with the ability to promote future chronic disease cost offsets through proactive patient behaviors. Incentives could be offered through tokenization for targeted patient groups. The ability to do this is not restricted to blockchain patient portals. EMR systems have this facility, although the form of incentives encompassed would not necessarily involve tokens but the more usual incentives of cash, vouchers or lottery tickets. Unfortunately, it is not clear that behavioral incentives influence behavior. The evidence for behavioral incentives is mixed and of low quality. The majority of evidence is from non-randomized observational studies with only a few well-conducted RCTs. There is no evidence, high quality or otherwise, for tokenized incentives within a blockchain environment. We have little idea of which incentives work best and at what level.

At the same time, any design intended to impact wellness or other proactive health behaviors has to recognize the possibility of perverse incentives. After all, the French in IndoChina in the 1920s attempted to reduce the rat population through bounties; the result being not a reduction in the rat population but the emergence of rat farms to increase supply.

\section{Management and Intermediation}

The potential for blockchains to be credible and to create value added outcomes will only be achieved if there is a management structure in place that supports intermediation. One way of looking at this is to think of the blockchain creating a managed market for data transfers and data aggregation. The blockchain owner could create this management structure or it could be subcontracted. The market brings together property owners (patients) as sellers with prospective third party clients for the data. Physicians may also participate in the value added information assembly (e.g., by generating reports to capture clinical endpoints).

Apart from ongoing technical support and marketing of the blockchain to prospective clients, successful intermediation in the managed market may require a substantial investment by blockchain owners in creating a technology assessment skill set. The skill set would need to embrace: (i) protocol developments and applications in target patient groups to evaluate claims; (ii) virtual file creation and de-identification to create exportable files; (iii) techniques for claims assessment using both aggregate data sets and micro-records; (iv) developing platforms for claims assessment to support disease area and therapeutic class reviews; and (vi) techniques and standards for instrument validation and instrument selection to track outcomes in disease areas.

A useful example of value added business service model utilizing EMRs is the Clinical Practice Research Datalink (CPRD) in the UK. Launched in April 2012, and building upon the earlier established General Practice Research Database (GPRD) the CPRD provides access to de-identified patient records linked to a range of data sets. The database includes demographics, diagnoses and symptoms, therapy interventions, outcomes of therapy, withdrawal correlates, referrals, laboratory tests and lifestyle factors (weight, alcohol use and smoking). To support clients, the CPRD staff offer services in pharmacoepidemiology, health technology assessment, protocol development and research study support.

The first task for any intermediation service would be to identify target patient groups from the blockchain patient base. If a third party wishes to identify patients by a specific ICD-10-CM code, then the blockchain must have a portal or window that allows patients with that characteristic to be identified prior to seeking permission to access the identified patient's EMR. It seems impractical (and inconsistent), given the firewalls in place to protect the data, to allow a 'backdoor' so that the intermediary can scan quickly the patient population to identify (i) the target patient group and (ii) solicit access permissions (with smart contracts) from the patient.. Irrespective of whether a 'search characteristics' portal is in place, the intermediary will still have to seek permissions from individual patients. This may involve a quota system where a target number of patients are selected (random selection with replacement?). If there are further sub-categories of patients there is a further level of search. This might apply, for example, if a claims assessment study is being proposed where patients are matched to the inclusion/exclusion criteria of a pivotal RCT.

\section{Enrollment and Uptake}

It is unlikely that patients will enroll in a blockchain product independently of support from their provider or health system. Patients would need to be aware of the blockchain, the benefits it offers as a personal healthcare record and the potential monetization incentives through allowing data access to third parties. The obvious 'recruiting agent' is the provider.

If the provider is the recruiting agent then the question arises: what incentives, if any, are needed to encourage recruitment. Providers would presumably have to spend time detailing the blockchain role as a secure healthcare record, the process by which data are transferred to the blockchain and how patients could monetize their data. Training programs may have to be offered providers so they are familiar, for example, with the mechanisms of cryptocurrency exchanges and tokenization.

The ability of an individual patient to monetize his/her health record will depend upon the ability of the blockchain to attract recruits. While there is no evidence to date to suggest what a minimum blockchain membership is required to ensure 
commercial viability, an 'all purpose' blockchain based on primary care would probably need in excess of 100,000 members to enable third parties to recruit target patient populations. This is dictated by the prevalence of specific disease states in the general population. More focused blockchains (or individual super nodes) are certainly possible (e.g., for cardiovascular patients) but the issue is still one of recruitment of a minimum number of patients through specialty provider practices.

\section{Property Rights}

If blockchains in healthcare are to achieve their promised potential, a fundamental requirement is that the patient has sole and exclusive rights to the healthcare data. Presently, there are few jurisdictions where patients are assured of the ownership of their health records. In the US, New Hampshire is the only state where these rights are legislated in favor of the patient. In other states legislation vests these rights in the provider with the majority having no legislative right assigned to either patient or provider. Patients have a 'right' to 'copies' of their health record. Whether this extends beyond the ability, for a fee, to obtain paper/fax copies of their record to electronic data transfer to a blockchain is an open question.

A recent commentary on the privacy and health data points to the unsatisfactory nature of data privacy and the limitation of safeguards established under the Health Insurance Portability and Accountability Act (HIPAA) $1996{ }^{5}$. The authors point to gaps and inconsistencies in health information privacy safeguards. Although HIPAA is intended to support data privacy in clinical settings, health insurance and research, internet health data are generally not covered. Companies can solicit sensitive health data on line, often with only superficial regard to the issues of privacy and access. These data, which all too often are not de-identified, can be sold to third parties for targeting individuals for direct solicitation and messaging. The key issue is that HIPAA, which compares unfavorably with European standards under the 2018 European Union General Data Protection Regulation, 'creates artificial distinctions between data generated in clinical or health insurance settings and online settings'.

A blockchain can protect health data, but moving data to a blockchain says nothing about the privacy of those data elements that are still held in the various electronic medical records. These data are still open to hacking with continuing concerns over the adequacy of firewalls in place to protect that information, and the extent to which breaches of patient confidentiality are monitored. Particularly concerning, are the potentially higher risks associated with claims for interoperability where a health system aims to link disparate providers and sites. Where there are claims for interoperability, are the transactions monitored, validated and encrypted not only to protect privacy but also the content of the transaction against subsequent modification or erasure?

Although outside of the scope of this commentary, a strong case can be made for revisiting HIPAA and expanding its scope to embrace both data generated within clinical, insurance and research settings and data generated through the internet. This would include not only requirements for transaction accounting with standards for consent, nomination of recipients and data collection technologies, but additional standards for 'offshore' data solicitation and other attempts to circumvent national data protection standards. Even with legislative change designed to protect data, there is no presumption that the patient 'owns' his/her data with attendant rights for determining access and transaction accounting. This should be a priority in legislation. The legislation should also ensure that there are transparent 'optin' provisions for patients not willing to move their records to a blockchain.

The issue of property rights and pushback by patients for the unsolicited use of their health records is shown in the current debate in Australia over the role of the Australian Digital Health Agency (ADHA). Following on from an earlier failed attempt to equip every Australian resident with an electronic health record, the ADHA is committed to establishing a lifetime health record (My Health Record) where property rights are retained by the government. Because of what was seen as a slow uptake (with, currently, only $25 \%$ of the target population enrolled) legislation was amended in December 2017 to switch participation from opting-in to the system to opting-out. Needless to say this is seen as a less than popular move given the ability of third parties to access these data over digital networks for research and other purposes without the permission of the patient. Currently, Australians have only until mid-October to opt out (presumably the unborn will not have this option). Also, it is noted that the current enrollment is an overstatement as it includes the opting-in by physicians who have supplied patient records for large scale trials.

Management of the ADHA was entrusted to a director who led the similar failed UK initiative (in England) 'Care Data'. The collapse of this initiative was seen as reflecting a failure to receive 'social approval' 6 . An assessment of the 'Care Data' failure emphasizes that 'legal authority' does not necessarily command social legitimacy. In other words legislative passage 'does not equate to a societal seal of approval or to securing the trust and confidence of patients, citizens, healthcare professional and researchers".

There are lessons here for blockchains: to succeed, even if legislation empowers patients through an unambiguous allocation of property rights, blockchains as private sector initiatives in health care, must gain public acceptance. They 
have to be seen as superior, not only to the numerous provider practice portals already in place which allow patients to view their health records, but in terms of a seamless integration of data, ease of access and recognized incentive structures. More to the point, adoption of blockchains by health systems have to be seen as a platform to support initiatives to improve outcomes of care and reduce direct medical cost. Although early days in the case for blockchains, the absence of pilot studies with credible claims is a hurdle to overcome. Can a blockchain owner, for example, demonstrate the applicability of blockchain platform based distributed applications in healthcare outcomes and resource allocation as technical and commercially viable products?

A final point: to date, the blockchain literature has focused on the platform supporting potential tokenization and monetization of individual health records. What is overlooked is the intermediation role of the provider. If applications are to drive monetization for participants then the role of the provider, as a participant in data assembly for applications, needs to be factored into the incentive structure.

\section{Overview}

The technical feasibility of a blockchain platform as a secure data depository, as a tamper-proof record of transactions, and as a basis for cryptocurrency exchange has long been established. What has yet to be established is the application of blockchain technology, not only as a platform enabling patient property rights and access to health data, but the potential for the blockchain to support a managed market for the monetization of health data to third parties for research and other activities. There are two prerequisites for a successful implementation of blockchain technology into heath care: (i) the unambiguous assignment of property rights of health data to the patient allied with an unimpeded access for transferring data to the blockchain; and (ii) an effective organizational structure and skill set that the blockchain vendor can demonstrate in supporting the managed market. Capturing data is one thing; the ability to respond to third party requests for data access to address specific research questions is another.

Conflicts of Interest: No conflicts were declared by either PCL or REM

\section{References}

${ }^{1}$ Innovations in Pharmacy. https://pubs.lib.umn.edu/index.php/innovations/section/view/formularyevaluations

${ }^{2}$ Langley PC. Information or Evidence? Abandoning imaginary worlds for blockchains in formulary decision making. Inov Pharm 2018;9(3):No 12

${ }^{3}$ Hanauer D, Wu D, Yang L et al. Development and empirical user-centered evaluation of semantically-based query recommendation for an electronic health record search engine. J Biomed Inform. 2017;67:1-10

${ }^{4}$ Langley PC. Langley PC. Nullius in Verba: Version 2.0 of the University of Minnesota, School of Social and Administrative Pharmacy Program, Proposed Guidelines for Formulary Evaluation. Inov Pharm. 2016;7(4): No 16

${ }^{5}$ Gostin O, Gostin J. Health data and privacy in the digital era. Viewpoint. JAMA. 2018;320:3

${ }^{6}$ Carter P, Laurie G, Dixon-Woods M. The social licence for research: why caredata ran into trouble. J Med Ethics. 2015;41:404-409 